\title{
Population Monotonicity in Public Good Economies with Single Dipped Preferences
}

\author{
İpek Gürsel Tapk1 ${ }^{1}$ \\ ${ }^{1}$ Faculty of Economics, Administrative and Social Sciences, Kadir Has University, Istanbul, Turkey \\ Correspondence: İpek Gürsel Tapk1, Faculty of Economics, Administrative and Social Sciences, Kadir Has \\ University, Istanbul, Turkey. E-mail: ipek.tapki@khas.edu.tr
}

Received: September 10, 2015

Accepted: February 6, 2016

Online Published: March 25, 2016

doi:10.5539/ijef.v8n4p80

URL: http://dx.doi.org/10.5539/ijef.v8n4p80

\begin{abstract}
We study public good economies with variable population. We consider the problem of locating a single public good along a segment when agents have single dipped preferences. We analyze population monotonicity along with the standard properties Pareto efficiency, continuity and no-veto power. We show that there is no rule satisfying these properties together.
\end{abstract}

Keywords: single-dipped preferences, population monotonity, variable population

\section{Introduction}

We study public good economies with single dipped preferences. Consider the problem of locating a public bad, like a dumping site. Each agent prefers locating it farther away from his or her home. The worst location for each agent is locating it right by their home. Such preferences are called single dipped preferences. There are many studies about thosepreferences, see Klaus (2001), Barbera, Berga, and Moreno (2009), and Manjunath (2013) etc.

A rule takes any such problem and it sets a location for the public bad. In this paper, we analyze population monotonicity requirement which is very standard in the literature. Consider a problem and suppose a rule sets the location of the public bad. For some reason, suppose a group of agents leave the society. Then, if a rule satisfies population monotonicity,then it should choose a location in which either all remaining agents are (weakly) better offor they are all (weakly) worse off than the initial location.

We show that there is no rule satisfying population monotonicity and other well-knownproperties: Pareto efficiency, no-veto power and continuity. Although these are very standardproperties in the literature, for our domain they are strong enough so that one cannot find a rule that satisfies all the mentioned properties.

Our paper is similar to Manjunath (2013). For the domain of single dipped preferences, he gives a characterization of strategy proof and unanimous rules. Barbera, Berga, and Moreno (2009) also study a similar problem. They also analyze strategy proof rules for theseproblems but they do not concentrate on a bounded interval for the location. Both papersconsider public good economies with a fixed population. We extend this model to a variablepopulation. This is the main difference between our model and theirs.

The paper is organized as follows. In Section 2, we introduce the model and in Section3, we analyze the implications of population monotonicity.

\section{Method}

In this paper, we theoretically analyze public good economies with single dipped preferences. We consider the implications of well-know axiomspopulation monotonicity, Pareto efficiency, continuity and no-veto power. Now, we will introduce our model and these axioms.

Let $\mathbb{N}$ be the set of potential agents and $\mathcal{N}$ be the collection of all finite subsets of $\mathbb{N}$. Let $A=[0, T]$ be the range of outcomes from which society must pick one. Let $\mathcal{B}$ denote the set of all non-empty closed subsets of A. A preference relation $R_{0}$ on $\mathrm{A}$ is single-dipped if there is a point $\mathrm{x}$ in $\mathrm{A}$ such that for each pair $\mathrm{y}$ and $\mathrm{z}$ in $\mathrm{A}$, if $\mathrm{z}$ $<\mathrm{y} \leq \mathrm{x}$ and $\mathrm{x}<\mathrm{y} \leq \mathrm{z}$, then $z P_{0} y$. The point $\mathrm{x}$, called the dip of $R$, is denoted by $\mathrm{d}(\mathrm{R})$. A preference relation $R_{0}$ on A is continuous if for each $i \in N$ and each $x \in A$, the sets $\left\{y \in A: y R_{i} x\right\}$ and $\left\{y \in A: x R_{i} y\right\}$ are closed. 
Each agent is equipped with a continuous and single-dipped preference relaton. Let $\mathcal{R}_{s d}$ be the set of such preferences and let $\mathcal{R} \equiv \mathrm{U}_{N \in \mathcal{N}} \mathcal{R}_{s d}^{N}$.

A choice function is a mapping $\varphi: \mathcal{R}_{s d} \times \mathcal{B} \rightarrow \mathcal{B}$ associating to each preference profile $R \in \mathcal{R}_{s d}$ and each $B \in \mathcal{B}$, an outcome $\varphi(R, B)$ in $\mathrm{B}$.

For each $R \in \mathcal{R}_{s d}^{N}$, let $N_{0}(R) \equiv\left\{i \in N: 0 P_{i} T\right\}, N_{T}(R) \equiv\left\{i \in N: T P_{i} 0\right\}$, and $N_{0 T}(R) \equiv\left\{i \in N: 0 I_{i} T\right\}$. Also, let $\bar{x}(R) \equiv \max _{i \in N_{0}(R)}\left\{x \in A: x I_{i} T\right.$ and $\left.x \leq d\left(R_{i}\right)\right\}$ and $\underline{x}(R) \equiv \min _{i \in N_{T}(R)}\left\{x \in A: x I_{i} 0\right.$ and $\left.x \geq d\left(R_{i}\right)\right\}$.

Let $(R, B) \in \mathcal{R}_{s d}^{N} \times \mathcal{B}$ and $x, y \in B$. Then, $\mathbf{x}$ Pareto dominates $\mathbf{y}$ at $\mathbf{R}$ if for each $i \in N, x R_{i} y$ and for at least one $j \in N, x P_{j} y$. An outcome $x \in B$ is (Pareto) efficientat $(\mathbf{R}, \mathbf{B})$ if there is no $y \in B$ that Pareto dominates $x$ at $(\mathrm{R}, \mathrm{B})$. Let $\mathbf{P O}(\mathbf{R}, \mathrm{B})$ denote the set of all efficient outcomes at $(\mathrm{R}, \mathrm{B})$.

We know that for each $R \in \mathcal{R}_{s d}^{N}$, the set of efficient outcomes at (R, A).

$$
P O(R, A)= \begin{cases}\{0\} & \text { if }\left|N_{0}(R)\right|>0 \text { and }\left|N_{T}(R)\right|=0, \\ \{T\} & \text { if }\left|N_{T}(R)\right|>0 \text { and }\left|N_{0}(R)\right|=0, \\ \{0, T\} & \text { if }\left|N_{0}(R)\right|>0,\left|N_{T}(R)\right|>0, \text { and } \underline{x}(R) \geq \bar{x}(R), \text { or } N_{0 T}=N . \\ \{0, T\} \cup(\underline{x}(R), \bar{x}(R)) & \text { if }\left|N_{0}(R)\right|>0,\left|N_{T}(R)\right|>0, \text { and } \underline{x}(R)<\bar{x}(R) .\end{cases}
$$

For any other closed subset $\mathrm{B}$ of $\mathrm{A}$, we have the following:

Lemma. For each $(R, B) \in \mathcal{R}_{s d}^{N} \times \mathcal{B}, P O(R, A) \cap B \subseteq P O(R, B)$. Moreoever, if $\{0, T\} \subseteq B$, then $P O(R, A) \cap A=P O(R, B)$.

Proof. Let $(R, B) \in \mathcal{R}_{s d}^{N} \times \mathcal{B}$ and $x \in P O(R, A) \cap B$. Then, no $y \in$ APareto dominates $x$. Thus, no $y \in B \subseteq$ A Pareto dominates $x$. Thus, $x \in P O(R, B)$. Now, let $\{0, T\} \subseteq B$ and $x \in P O(R, B)$. Suppose, by contradiction, that $x \notin P O(R, A)$. Then, there is $y \in A \backslash B$ that Pareto dominates $x$. But, then by the definition of single-dipped preferences, either 0 or T Pareto dominates $x$, a contradiction to $x \in P O(R, B)$.

We will analyze the following properties of a choice function:

Pareto efficiency: For each $N \in \mathcal{N}$ and each $(R, B) \in \mathcal{R}_{s d}^{N} \times \mathcal{B}, \varphi(R, B) \in P O(R, B)$.

Now, let $(R, B) \in \mathcal{R}_{s d}^{N} \times \mathcal{B}$ and $x \equiv \varphi(R, B)$. Suppose that a group of agents leave. Then, after this change, if $\varphi$ is population monotonic, it chooses a point in which either all remaining agents are (weakly) better off or they are all (weakly) worse off than $x$. For a detailed discussion of this property, see Thomson (1983), Ching and Thomson (1993).

Population Monotonicity: For each pair $N, N^{\prime} \in \mathcal{N}$ with $N^{\prime} \subseteq N$, each $R \in \mathcal{R}_{s d}^{N}$, each $R^{\prime} \in \mathcal{R}_{s d}^{N}$ with $R^{\prime}=R_{N^{\prime}}$, and each $B \in \mathcal{B}$, if $x \equiv \varphi(R, B)$, then either [for each $i \in N^{\prime}, y R^{\prime}{ }_{i} x$ ] or [for each $i \in N^{\prime}, x R_{i}^{\prime} y$ ].

Now, let $(R, B) \in \mathcal{R}_{s d}^{N} \times \mathcal{B}$. Suppose that an alternative, $x$ in $\mathrm{B}$ is top ranked with respect to $\mathrm{R}$ by all agents except possibly one. Then, if $\varphi$ satisfies no veto-power, $x$ should be chosen for (R, B).

No Veto-Power: For each $(R, B) \in \mathcal{R}_{s d}^{N} \times \mathcal{B}$ and each $x \in B$, if for each $i \in N \backslash\{j\}$ and each $y \in B \backslash\{x\}$, we have $x P_{i} y$, then $\varphi(R, B)=x$.

Let $R_{i} \in \mathcal{R}_{s d}$ and $x \in A$. Let $A_{R_{i}}(x)=\left\{y \in A: y \geq d\left(R_{i}\right)\right.$ if $x \leq d\left(R_{i}\right)$, and $y \leq d\left(R_{i}\right)$ if $\left.x \geq d\left(R_{i}\right)\right\}$. The equivalent point of $x$ under $R_{i}, e_{R_{i}}(x)$ is given by the following two conditions: (i) $e_{R_{i}}(x) \in A_{R_{i}}(x)$, (ii) there is no $y \in A_{R_{i}}(x)$ such that $e_{R_{i}}(x) P_{i} y R_{i} x$. Note that, for each $R_{i} \in \mathcal{R}_{s d}$ and each $x \in A, e_{R_{i}}(x)$ exists and it is unique. The distance between two preference relations $R_{1}, R_{2} \in \mathcal{R}_{s d}$ is defined as follows:

$$
d\left(R_{1}, R_{2}\right) \equiv \max _{x \in A}\left|e_{R_{1}}(x)-e_{R_{2}}(x)\right|
$$

Since preferences are continuous, $e_{R_{1}}$ and $e_{R_{2}}$ are continuous functions. Thus, $d\left(R_{1}, R_{2}\right)$ is continuous. 
Continuity: For each pair $R, R^{\prime} \in \mathcal{R}_{s d}$ and each $\mathcal{E}>0$, there is $\delta>0$ such that if $0<d\left(R, R^{\prime}\right)<\delta$, then $\left|\varphi(R, A)-\varphi\left(R^{\prime}, A\right)\right|<\varepsilon$.

The followings are examples of choice functions. Given a problem, the choice function in Example 1 chooses the minimal Pareto efficient outcome at that problem, and the choice function in Example 2 chooses the maximal one.

Example 1. For each $N \in \mathcal{N}$ and each $(R, B) \in \mathcal{R}_{s d}^{N} \times \mathcal{B}$,

$$
\varphi^{\min }(R, B) \equiv \min _{x \in P O(R, B)} x
$$

Note that, $\varphi^{\min }$ satisfies Pareto efficiency and population monotonicity but neither continuity nor no veto-power.

Example 2. For each $N \in \mathcal{N}$ and each $(R, B) \in \mathcal{R}_{s d}^{N} \times \mathcal{B}$,

$$
\varphi^{\max }(R, B) \equiv \max _{x \in P O(R, B)} x
$$

Note that $\varphi^{\max }$ satisfies Pareto efficiency and population monotonicity but neither continuity nor no veto-power. Now, let $>=\left(>^{N}\right)_{N \in \mathcal{N}}$ be a family of orderings. For simplicity, for each $N \in \mathcal{N}$, let $>^{N}$ be the natural ordering. Given any society N, the following function chooses the most preferred outcome for 1 , if there is only one such alternative. If not, it chooses the most preferred outcome for 2 , if there is only one and so on. If all agents have more than one most preferred outcome, then it chooses the minimal outcome. Formally,

Example 3. For each $B \in \mathcal{B}$, lete $e^{B}=\min _{x \in B} x$. For each $(R, B) \in \mathcal{R}_{s d}^{N} \times \mathcal{B}$, define the serial dictatorship rule with respect to $>$ as

Note that, for each $>, \boldsymbol{D}^{\succ}$ is Pareto efficient. It violates population monotonicity, continuity and no veto-power.

\section{Results}

The choice functions in Examples 1, 2, and 3 do not satisfy Pareto efficiency, population monotonicity, continuity and no veto-power together. In fact, this is not specific to these examples of choice functions. We first show that there is no continuous choice function that is also Pareto efficient.

Theorem 1. No choice function satisfies Pareto efficiency and continuity.

Proof. Suppose $\varphi$ satisfies Pareto efficiency. Let $N=\{1,2\}$. Let $R_{1}, R_{2} \in \mathcal{R}_{\text {sd }}$ be such that $N=N_{0 T}(R)$. Then, $P O(R, B)=\{0, T\}$. Since $\varphi$ satisfies Pareto efficiency, $\varphi(R, B) \in\{0, T\}$. First, suppose $\varphi(R, B)=0$. Let $\left\{R^{v}\right\}$ be a sequence of preference relations in $\mathcal{R}_{s d}$ such that for each $v \in \mathbb{N}, T P^{v} 0$ and $d\left(R^{v}, R_{1}\right)<\delta$. Then, for each $v \in \mathbb{N}, P O\left(R^{v}, R_{2}, B\right)=\{T\}$. Since $\varphi$ is Pareto efficient, for each $v \in \mathbb{N}, \varphi\left(R^{v}, R_{2}, B\right)=T$. Thus, $\varphi$ is not continuous. Now, suppose $\varphi(R, B)=T$. Let $\left\{R^{v}\right\}$ be a sequence of preference relations in $\mathcal{R}_{\text {sd }}$ such that for each $v \in \mathbb{N}, 0 P^{v} T$ and $d\left(R^{v}, R_{1}\right)<\delta$. Then, for each $v \in \mathbb{N}, P O\left(R^{v}, R_{2}, B\right)=\{0\}$. Since $\varphi$ is Pareto efficient, for each $v \in \mathbb{N}, \varphi\left(R^{v}, R_{2}, B\right)=0$. Thus, $\varphi$ is not continuous.

The next theorem analyzes the implications of Pareto efficiency, population monotonicity and no veto-power.

Theorem 2. No choice function satisfies Pareto efficiency, population monotonicity and no veto-power.

Proof. Suppose $\varphi$ satisfies Pareto efficiency and no veto-power. Let $\mathrm{N}=\{1,2,3,4\}$. Let $(R, B) \in \mathcal{R}_{s d}^{N} \times \mathcal{B}$ be such that $N_{0}(R)=\{1,2\}, N_{T}(R)=\{3,4\}$ and $P O(R, B)=\{0, T\}$. Then, no veto-power implies that $\varphi\left(R_{1}, R_{2}, R_{3}, B\right)=0$ and $\varphi\left(R_{1}, R_{3}, R_{4}, B\right)=\{T\}$. Pareto efficiency implies $\varphi(R, B) \in\{0, T$. Suppose $\varphi(R, B)=0$. Then, note that $\varphi(R, B) P_{1} \varphi\left(R_{1}, R_{3}, R_{4}, B\right)$ and $\varphi\left(R_{1}, R_{3}, R_{4}, B\right) P_{3} \varphi(R, B)$. Thus, $\varphi$ is not population monotonic. Now, suppose $\varphi(R, B)=T$. Then, note that $\varphi\left(R_{1}, R_{2}, R_{3}, B\right) P_{1} \varphi(R, B)$ and $\varphi(R, B) P_{3} \varphi\left(R_{1}, R_{2}, R_{3}, B\right)$. Thus $\varphi$ is not population monotonic.

\section{Discussion}

We study public good economies with single dipped preferences. We show that there is no rule that satisfies population monotonicity together with Pareto efficiency, continuity and no veto-power. Although these are standard properties in many different contexts in the literature, in our model, they are very strong. 


\section{References}

Barbera, S., Berga, D., \& Moreno, B. (2009). Single-dipped preferences. Barcelona Economic Working Paper Series, 418.

Klaus, B. (2001). Population-monotonicity and separability for economies with single-dipped preferences and the assignment of an indivisible object. Economic Theory, 17, 675-692. http://dx.doi.org/10.1007/PL00004123

Manjunath, V. (2013). Efficient and Strategy-proof Social Choice When Preferences are Single-dipped. mimeo.

Thomson, W., \& Ching, S. (1995). Population Monotonic Solutions in Public Good Economies with Single Peaked Preferences. University of Rochester Working Paper.

Yeh, C. H. (2006). Reduction-consistency in Collective Choice Problems. Journal of Mathematical Economics, 42(6), 637-652. http://dx.doi.org/10.1016/j.jmateco.2005.12.002

Young, H. P. (1975). Social Choice Scoring Functions. SIAM Journal of Applies Mathematics, 28, 824-838. http://dx.doi.org/10.1137/0128067

\section{Copyrights}

Copyright for this article is retained by the author(s), with first publication rights granted to the journal.

This is an open-access article distributed under the terms and conditions of the Creative Commons Attribution license (http://creativecommons.org/licenses/by/3.0/). 